Experimental validation of a method for removing the capacitive leakage artifact from electrical bioimpedance spectroscopy measurements

This article has been downloaded from IOPscience. Please scroll down to see the full text article.

2010 Meas. Sci. Technol. 21115802

(http://iopscience.iop.org/0957-0233/21/11/115802)

View the table of contents for this issue, or go to the journal homepage for more

Download details:

IP Address: 109.58.91.168

The article was downloaded on 07/10/2010 at 06:09

Please note that terms and conditions apply. 


\title{
Experimental validation of a method for removing the capacitive leakage artifact from electrical bioimpedance spectroscopy measurements
}

\author{
R Buendia ${ }^{1,2}$, F Seoane $^{1,3}$ and R Gil-Pita ${ }^{2}$ \\ ${ }^{1}$ School of Engineering, University of Borås, SE-501 90 Borås, Sweden \\ 2 Department of Signal Theory and Communications, University of Alcala, ES-28871, Madrid, Spain \\ ${ }^{3}$ Department of Signal \& Systems, Chalmers University of Technology, SE-41296, Gothenburg, Sweden \\ E-mail: ruben.buendia@hb.se
}

Received 8 June 2010, in final form 6 September 2010

Published 6 October 2010

Online at stacks.iop.org/MST/21/115802

\begin{abstract}
Often when performing electrical bioimpedance (EBI) spectroscopy measurements, the obtained EBI data present a hook-like deviation, which is most noticeable at high frequencies in the impedance plane. The deviation is due to a capacitive leakage effect caused by the presence of stray capacitances. In addition to the data deviation being remarkably noticeable at high frequencies in the phase and the reactance spectra, the measured EBI is also altered in the resistance and the modulus. If this EBI data deviation is not properly removed, it interferes with subsequent data analysis processes, especially with Cole model-based analyses. In other words, to perform any accurate analysis of the EBI spectroscopy data, the hook deviation must be properly removed. Td compensation is a method used to compensate the hook deviation present in EBI data; it consists of multiplying the obtained spectrum, $Z_{\text {meas }}(\omega)$, by a complex exponential in the form of $\exp (-\mathrm{j} \omega \mathrm{Td})$. Although the method is well known and accepted, Td compensation cannot entirely correct the hook-like deviation; moreover, it lacks solid scientific grounds. In this work, the Td compensation method is revisited, and it is shown that it should not be used to correct the effect of a capacitive leakage; furthermore, a more developed approach for correcting the hook deviation caused by the capacitive leakage is proposed. The method includes a novel correcting expression and a process for selecting the proper values of expressions that are complex and frequency dependent. The correctness of the novel method is validated with the experimental data obtained from measurements from three different EBI applications. The obtained results confirm the sufficiency and feasibility of the correcting method.
\end{abstract}

Keywords: electrical bioimpedance spectroscopy, capacitive leakage, artifact removal (Some figures in this article are in colour only in the electronic version)

\section{Introduction}

Electrical bioimpedance (EBI) spectroscopy is a typical approach currently employed in several applications of EBI analysis, such as total body composition assessment (Moissl et al 2006), electronic biopsies of skin tissue (Aberg et al 2004, 2005) and detection of pulmonary edema (Beckmann et al 2007).

To perform any useful data analyses, in addition to using an appropriate analysis method, the data should be free from interferences or artifacts. It is rather common to 
obtain EBI measurements that are affected by the capacitive leakage caused by parasitic capacitances in parallel with the measurement load (Bolton et al 1998, Mirtaheri et al 2007). The presence of such stray parasitic capacitances creates a characteristic EBI data deviation that is especially noticeable at high frequencies (Bolton et al 1998, Buendia 2009, Scharfetter et al 1998, van Marken Lichtenbelt et al 1994). Such a deviation is a capacitive leakage effect and is commonly known as hook or tail effect because it reassembles a hook when the impedance spectrum is presented on an impedance plot.

Originally, the capacitive leakage effect was ascribed to time delays associated with the measurement leads, and this premise was used as the basis for schemes to compensate for and remove the deviation (De Lorenzo et al 1997). However, according to several authors (Bolton et al 1998, Buendia 2009, Scharfetter et al 1998), the origin of the aforementioned hook-like deviation is related to parasitic stray capacitances. While differences on time delays only modify the phase, leaving the magnitude of the impedance unaltered, capacitive leakage affects both the real and the imaginary parts of the impedance, i.e. the whole complex EBI is altered-both the phase and the modulus. It is precisely the deviation observed in the modulus of the impedance measurement that points to capacitive leakage rather than time delay as the origin of the hook-like data deviation (Bolton et al 1998, Scharfetter et al 1998).

Although capacitive leakage modifies the impedance spectra more noticeably at high frequencies, the deviation of the complex EBI data is present at any ac frequency. This deviation in the EBI data interferes in any post hoc EBI data analysis, especially when a Cole-based analysis is required and when corrupted data that contain contributions from two dominant dispersions are attempted to be fitted into a single dispersion system, namely the Cole function (Cole 1940).

Currently, despite its limitations, the method of choice for correcting the influence of the capacitive leakage effect on EBI data is the so-called Td compensation (De Lorenzo et al 1997), which consists of fitting the EBI data to the Cole extended model (Scharfetter et al 1998). In this work, we wish to confirm the hypothesis postulated by Bolton et al (1998) and Scharfetter et al (1998) that the origin of the hook-like deviation in the EBI data is due to capacitive leakage and to present a solid method for correcting the measurement artifact that clearly overcomes the limitations of the Td compensation.

\section{Materials and methods}

\subsection{Theoretical and experimental validation}

To lay the foundation to obtain the right analytical solution for compensating or even canceling completely the influence of capacitive leakage on EBI measurements, a theoretical analysis of the effect of capacitive leakage on EBI measurements has been performed on a well-spread and accepted equivalent model with the software packages Mathematica and Matlab. The impedance load of the tissue under study (TUS) used in the equivalent model was modeled with a Cole function with values $R_{0}=449.6 \Omega, R_{\infty}=296.7 \Omega$,
Table 1. Electrode placement for the EBI measurements.

\begin{tabular}{lllll}
\hline$I+$ & $V+$ & $V-$ & $I-$ & Measurement type \\
\hline A & B & C & D & Right side \\
E & F & G & H & Segmental trunk \\
I & J & K & L & Segmental arm \\
\hline
\end{tabular}

$\tau=5.27 \times 10^{-6} \mathrm{~s}$ and $\alpha=0.7$. The Cole function was introduced by K S Cole in 1940 to fit EBI measurements, and it uses four parameters to reproduce EBI data on a single dispersion (Schwan 1957). The expression for the Cole function is represented in equation (1), where $R_{0}$ is the resistance at zero frequency, $R_{\infty}$ is the resistance at infinite frequency, $\tau$ is the time constant associated with the natural characteristic frequency, $\tau=\left(\omega_{C}\right)^{-1}$, and $\alpha$ is a factor to account for the difference in electrically polarizable elements constituting the TUS.

$$
Z_{\mathrm{COLE}}=R_{\infty}+\frac{R_{0}-R_{\infty}}{1+(\mathrm{j} \omega \tau)^{\alpha}}
$$

To experimentally validate the correctness of the proposed correcting method, experimental EBI measurements containing the well-known hook-like deviation at high frequencies have been corrected in Matlab with the proposed method.

\subsection{EBI measurements}

To validate the proposed correcting method, tetrapolar EBI measurements for body composition analysis (BCA) were taken from healthy subjects. The complex EBI measurements were obtained over the frequency range 3.096-1000 kHz with an SFB7 spectrometer manufactured by Impedimed using a measurement current with a constant rms amplitude of $200 \mu \mathrm{A}$. The SFB7 estimates the impedance of the TUS by sensing the voltage drop in the load caused by the injected current together with the actual current produced by the current source. In this way, measurement errors caused by the limitations of the current source, such as finite output impedance or frequency dependence of the output, are avoided.

The electrodes used for the EBI measurements were typical $\mathrm{Ag} / \mathrm{AgCl}$ repositionable Red Dot electrodes manufactured by $3 \mathrm{M}$ and were placed in typical locations for total right side, trunk segmental and arm segmental measurements, as shown in figure 1 and table 1 .

2.2.1. Total body right side. A total of $30 \mathrm{EBI}$ measurements were taken using the standard placement of electrodes on the hand, wrist, ankle and foot (points A, B, C and D in the figure) as shown in figure 1 (Kyle et al 2004). The test subject was a 24 year old male who was $185 \mathrm{~cm}$ tall and weighed $82 \mathrm{~kg}$.

2.2.2. Segmental trunk. A total of 100 EBI measurements were obtained with a tetrapolar electrode placement, as shown in figure 1 . The test subject was a 24 year old male who was $173 \mathrm{~cm}$ tall and weighed $79 \mathrm{~kg}$.

2.2.3. Segmental arm. A total of 100 EBI measurements were obtained with a tetrapolar wrist-to-shoulder electrode 


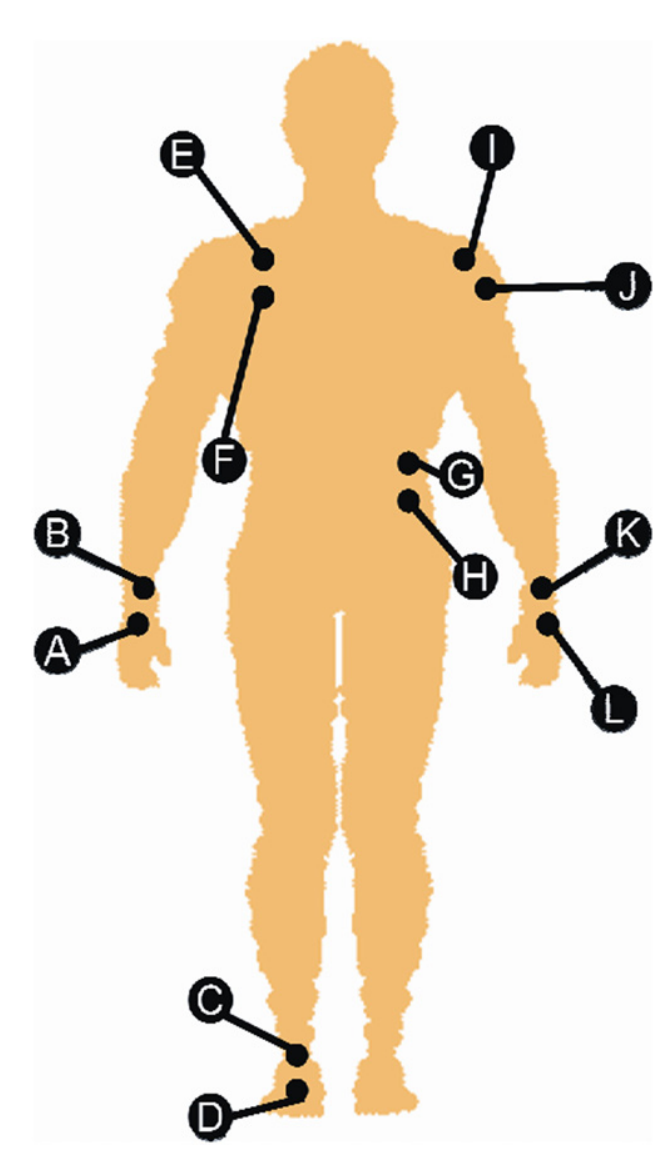

Figure 1. Diagram indicating the three tetrapolar electrode placements used for each of the performed EBI measurements.

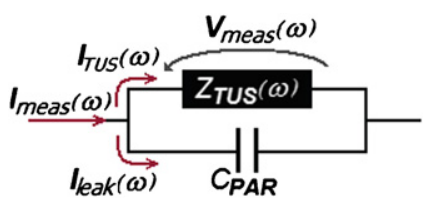

Figure 2. Model of study.

placement, as shown in figure 1 . The test subject was a 24 year old male who was $173 \mathrm{~cm}$ tall and weighed $79 \mathrm{~kg}$.

\section{Analysis}

\subsection{Capacitive leakage model analysis}

Simplifying the equivalent models for EBI measurements proposed by Scharfetter et al (1998) for the impedance and by Mirtaheri et al (2004) for the admittance, it is possible to obtain a simple equivalent electrical model (Buendia et al 2010). The obtained model is just a capacitive current divider, as shown in figure 2 .

\subsection{Hook-like artifact: origin and deviation of the EBI spectrum}

According to the equivalent models proposed in Scharfetter et al (1998), Ivorra (2005), Mirtaheri et al (2007) and Mirtaheri et al (2004), and the model depicted in figure 2, the electrical current intended for stimulating the measurement object, the TUS in this case, leaks away from the measurement load through parallel electrical pathways caused by parasitic capacitances. Such a current leakage introduces an impedance estimation error that is frequency dependent (Buendia et al 2010). Note that from figure 2 and equation (2), it is easy to realize that as long as there is an alternative parasitic pathway, part of the measurement current $I_{\text {meas }}(\omega)$ will not flow through $Z_{\mathrm{TUS}}(\omega)$. Therefore, the actual stimulating current $I_{\mathrm{TUS}}(\omega)$ will be smaller than the intended sensed current $I_{\text {meas }}(\omega)$. Thus, when applying Ohm's law to the sensed voltage $V_{\text {meas }}(\omega)$ that is caused by $I_{\mathrm{TUS}}(\omega)$ and not by the sensed $I_{\text {meas }}(\omega)$, the impedance $Z_{\mathrm{TUS}}(\omega)$ will be underestimated.

$$
Z_{\text {meas }}(\omega)=\frac{V_{\text {meas }}(\omega)}{I_{\text {meas }}(\omega)}=\frac{I_{\mathrm{TUS}}(\omega) \cdot Z_{\mathrm{TUS}}(\omega)}{I_{\mathrm{TUS}}(\omega)+I_{\text {leak }}(\omega)}
$$

The impedance estimation error caused by capacitive leakage depends on the amount of current that leaks away through the parasitic pathways $I_{\text {leak }}(\omega)$. Because $I_{\text {leak }}(\omega)$ flows through capacitive pathways, the value of $I_{\text {leak }}(\omega)$ increases with frequency, and consequently the estimation error is larger at higher frequencies than at lower frequencies. The frequency dependence of the estimation error depends on the impedance values of the branches of the capacitive current divider, which are dependent on the specific EBI application and the parasitic capacitances present in a specific measurement setup.

As shown in figure 3, the resulting impedance estimation error produces a deviation in the complex impedance spectrum. Although the deviation is especially noticeable in the spectra of both the reactance and phase at high frequencies, the produced deviation actually affects the EBI spectrum at all ac frequencies, altering both the real and imaginary parts of the EBI spectrum. This result means that both the modulus and phase of the EBI spectra are affected.

\subsection{Td compensation}

Td compensation is a well-known approach used for more than a decade for correcting the hook effect that consists of multiplying the measured EBI spectra by a complex exponential in the form of $\exp (-\mathrm{j} \omega \mathrm{Td})$. The $\mathrm{Td}$ term is considered to be a time delay.

Scharfetter et al (1998) and Buendia et al (2010) showed that multiplying any complex EBI spectrum by $\exp (-\mathrm{j} \omega \mathrm{Td})$ with a Td term only modifies the phase. Therefore, such an approach can only compensate for a deviation occurring in the phase and is completely unable to compensate for any estimation error produced over the modulus of the impedance. Bolton et al suggested in 1998 that the Td compensation method applied in the form of a complex exponential like $\exp (-\mathrm{j} \omega \mathrm{Td})$ was not the proper manner to correct the observed high-frequency data deviation (Bolton et al 1998). In that work, Bolton et al proposed a slightly more developed version of the $\mathrm{Td}$ compensation method, as can be observed in equation (3), the term Td remains, but the complex exponential disappears.

$$
Z_{\text {Corr }}(\omega)=Z_{\text {meas }}(\omega)\left(\frac{1}{1+\mathrm{j} \omega \mathrm{Td}}\right)
$$




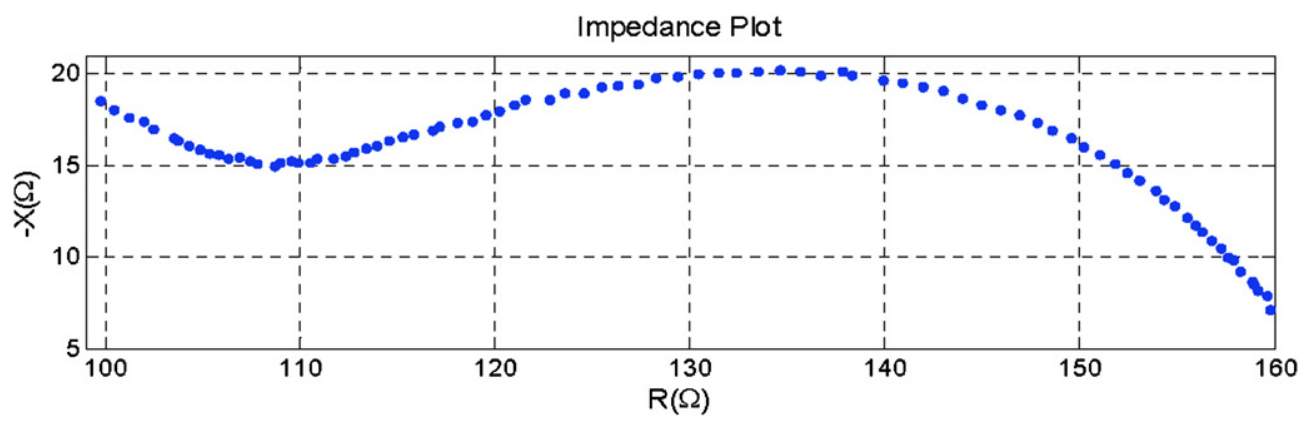

Figure 3. EBI data plotted in the impedance plane, from 3.096 to $1000 \mathrm{kHz}$, exhibiting a remarkable hook-like artifact.

\subsection{Full correction}

Analyzing the model presented in figure 2, one can show that to completely correct the impedance estimation error caused by the capacitive leakage with Td compensation, the value of $\mathrm{Td}$ in the complex exponential $\exp (-\mathrm{j} \omega \mathrm{Td})$ should be complex and a function of the frequency instead of just a single scalar. A newly developed method is proposed together with a procedure to obtain the values of the correction function (Buendia et al 2010).

\section{Proposed method}

The theoretical analysis of the Td compensation indicates that a full correction of the capacitive leakage effect can be achieved only if the measurement is multiplied by a complex exponential in the form $\exp (-\mathrm{j} \omega \mathrm{TD}(\omega))$, where $\operatorname{TD}(\omega)$ is a complex function of frequency. Note that the nomenclature $\operatorname{TD}(\omega)$ has been chosen to support the explanation, facilitating comparison with the expressions for Td compensation and equation (3). As shown in equation (4), the obtained analytical expression is a logarithmic function and depends on both the measured impedance $Z_{\text {meas }}(\omega)$ and the value of the parasitic capacitance $C_{\mathrm{PAR}}$. Developing the correcting expression in equation (5) further and with an efficient methodology for estimating the parasitic capacitance contained in the EBI measurements, a new artifact-removal method is introduced below to fully correct the EBI measurements contaminated with capacitive leakage effects.

\subsection{Correction function}

Substituting equation (4) in $\exp (-\mathrm{j} \omega \mathrm{TD}(\omega))$ cancels the complex exponential, leaving the correcting expression in equation (5). The obtained correcting expression is very similar to the expression proposed by Bolton et al (1998). The only difference in equation (5) is that $\mathrm{Td}$ is replaced by a complex function dependent on the measured impedance $Z_{\text {meas }}(\omega)$ and an estimated parasitic capacitance $C_{\text {PAR }}$ instead of taking the value of a single scalar as in equation (3).

$$
\begin{aligned}
\operatorname{TD}(\omega) & =\frac{\log \left[1-\mathrm{j} \omega Z_{\text {meas }}(\omega) C_{\text {PAR }}\right]}{\mathrm{j} \omega} \\
Z_{\text {Corr }}(\omega) & =Z_{\text {meas }}(\omega) \frac{1}{1-\mathrm{j} \omega Z_{\text {meas }}(\omega) C_{\text {PAR }}}
\end{aligned}
$$

\subsection{Parasitic capacitance estimation}

The electrical susceptance of a single dispersion system like any Cole-modeled tissue decreases toward zero after reaching its maximum value in a similar manner as the reactance does. When there is a capacitance in parallel with the TUS, the susceptance will increase instead of decreasing with frequency; see figure 4 . At frequencies where the susceptance of the TUS can be neglected, the increase of the susceptance with frequency will be set by the value of the parallel capacitance. The susceptance spectrum will then exhibit a slope that can be used to estimate the value of the capacitance, as indicated in figure 4.

\section{Validation results}

\subsection{Theoretical validation of the proposed correcting approach}

Figure 5 shows the correction effect caused by applying the correcting expression in equation (5) to $Z_{\text {meas }}(\omega)$ calculated with the model from figure 2 for a Cole-based impedance obtained with equation (1) and a parasitic capacitance of $50 \mathrm{pF}$. In the plots, especially in the reactance plot in figure $5(B)$, it can be observed how the hook deviation is completely removed to obtain $Z_{\text {Corr }}(\omega)$ identical to the original $Z_{\text {meas }}(\omega)$.

\subsection{Experimental validation of the approach}

The EBI spectroscopy measurements taken in the frequency range between $3.096 \mathrm{kHz}$ and $1000 \mathrm{kHz}$ containing capacitive leakage artifacts have been processed with the proposed artifact-removal method. The corrected results and the estimated parasitic capacitance are presented in the following sections.

5.2.1. Right side measurements. The plots in figures $6(A)-$ (C) contain the immittance data from one of the 30 EBI measurements obtained with the right side electrode placement. The experimental data are plotted with circular markers, and the corrected data are plotted with asterisks. The hook deviation is more noticeable in the reactance and the susceptance spectra in figures $6(B)$ and $(C)$, respectively. It can be seen from the plots that after the correction, the hook deviation has disappeared, leaving a typical single dominant 


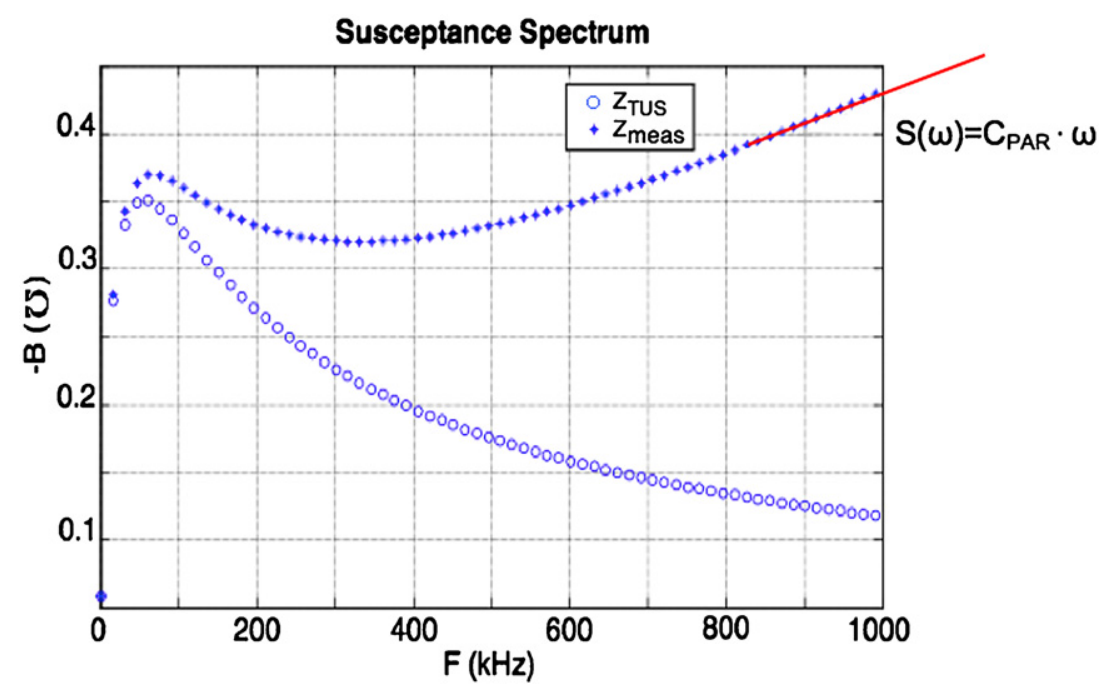

Figure 4. Susceptance of a wrist-to-ankle measurement with and without a $50 \mathrm{pF}$ capacitance in parallel. The immittance is modeled as a typical Cole single dominant dispersion.
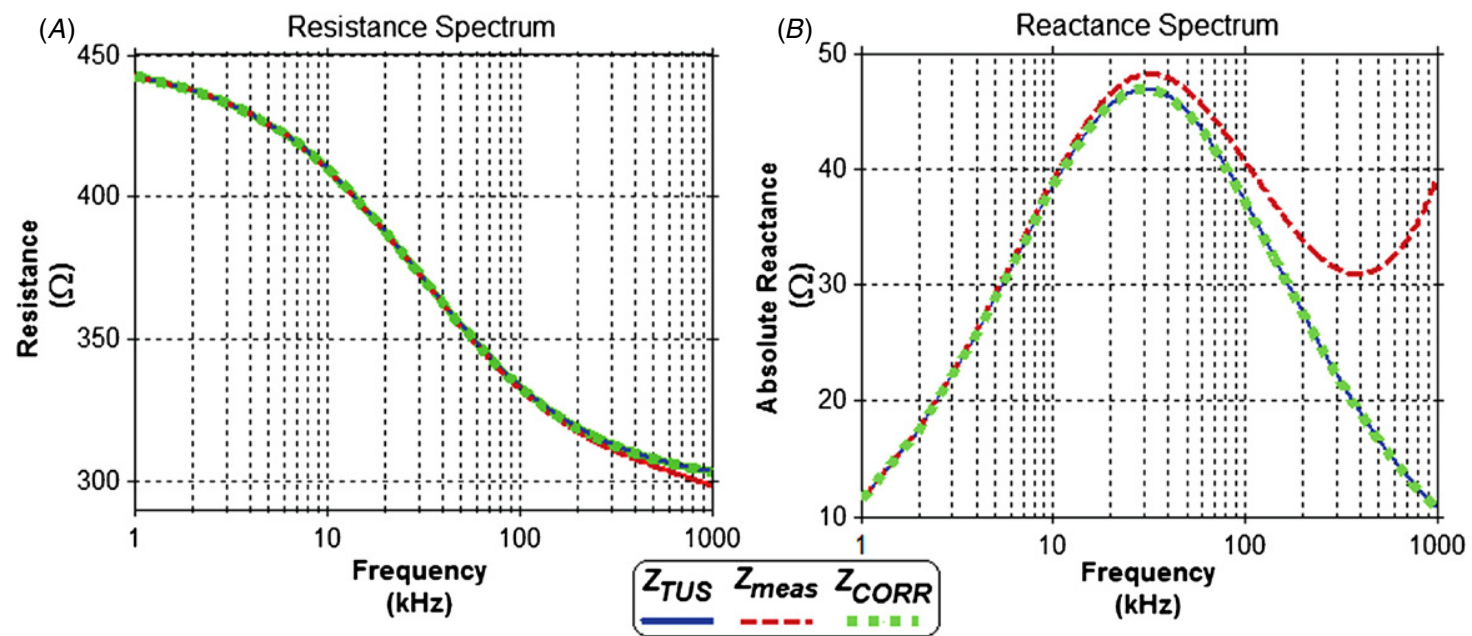

Figure 5. Correction effect of $F_{\text {Corr }}(\omega)$ over $Z_{\text {meas }}(\omega)$ with $C_{\mathrm{PAR}}$ of $50 \mathrm{pF}$.

dispersion. In figure $6(D)$, the parasitic capacitance estimated by the proposed correction method for all 30 measurements is plotted. As indicated in figure $6(D)$, the average estimated value for the parasitic capacitance was $40.94 \mathrm{pF}$ with a standard deviation of only $1.9 \mathrm{pF}$.

\subsubsection{Segmental arm measurements. The plots in} figures $7(A)-(C)$ contain the immittance data from one of the 100 EBI measurements obtained with the segmental arm electrode placement. The experimental and the corrected data are plotted with circular and asterisk markers, respectively. The capacitive leakage effect is noticeable in the impedance plot in figure $7(A)$, but the hook-like deviation can be clearly noticed in the reactance and the susceptance spectra in figures $7(B)$ and $(C)$, respectively. Once again, it is possible to observe in all three impedance and spectral plots that after the correction, the hook-like deviation has completely disappeared, leaving a typical single dominant dispersion. Figure $7(D)$ plots the parasitic capacitance estimated by the proposed correction method for the 100 measurements. As indicated in figure $7(D)$, the average estimated value for the parasitic capacitance was $120.29 \mathrm{pF}$ with a standard deviation of only $1.9 \mathrm{pF}$.

5.2.3. Segmental trunk measurements. The plots in figures $8(A)-(C)$ contain the immittance data from one of the $100 \mathrm{EBI}$ measurements obtained with the segmental trunk electrode placement. The experimental data are plotted with circular markers and the corrected data with asterisks. The hook-like deviation is easy to identify in all three plots: the impedance plot in figure $8(A)$, the reactance spectrum in figure $8(B)$ and the susceptance spectrum in figure $8(C)$. After the correction, it is possible to observe that the hook deviation has disappeared in all three plots. The corrected data represent a typical single dominant dispersion system. In figure $8(D)$, the parasitic capacitance estimated by the proposed correction method for all the measurements is plotted. As indicated in figure $8(D)$, the average value for the estimated parasitic capacitance was $683.23 \mathrm{pF}$ with a standard deviation of only $26.08 \mathrm{pF}$. 
(A)

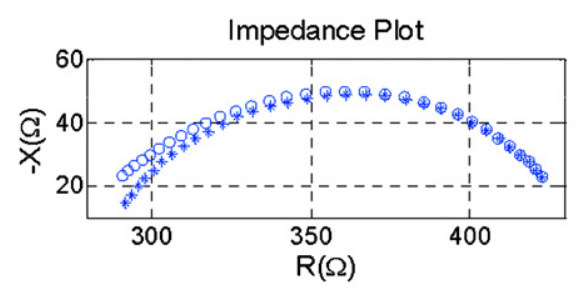

$(C)$

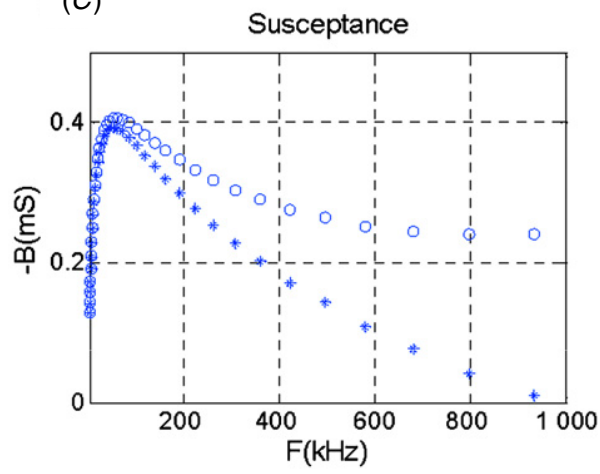

(B)

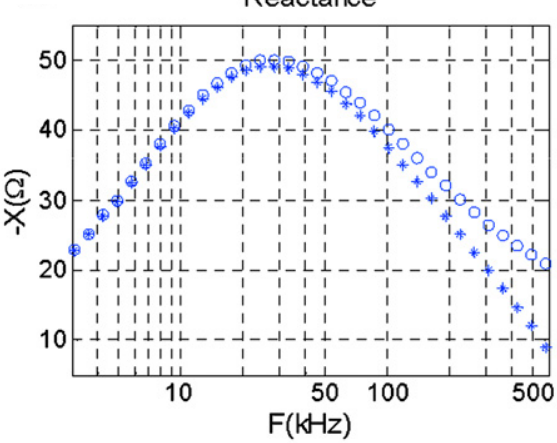

$(D)$

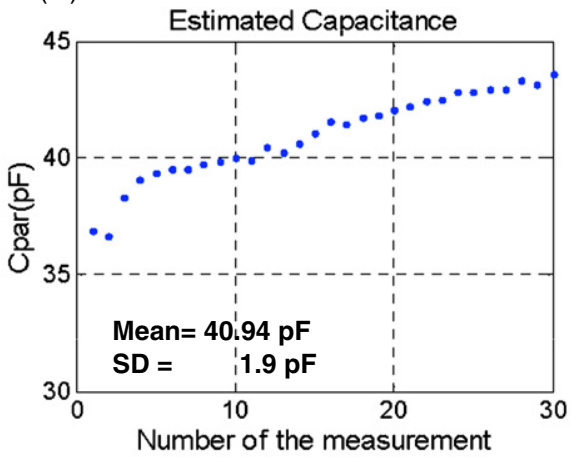

\section{- Experimental Measurement $\quad *$ Corrected Measurement $\quad$ Estimated Capacitance}

Figure 6. Experimental results obtained from the right side EBI measurements. Note that the mean and standard deviation values for the estimated capacitance are annotated in $(D)$.

$(A)$

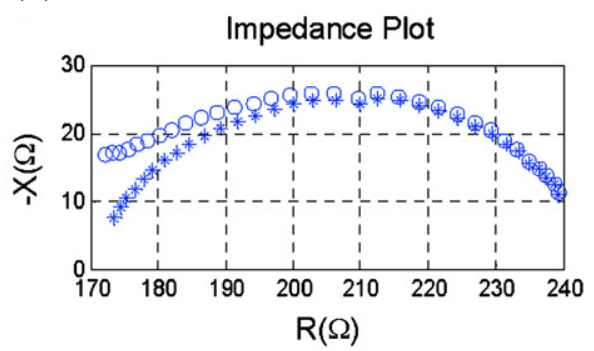

$(C)$

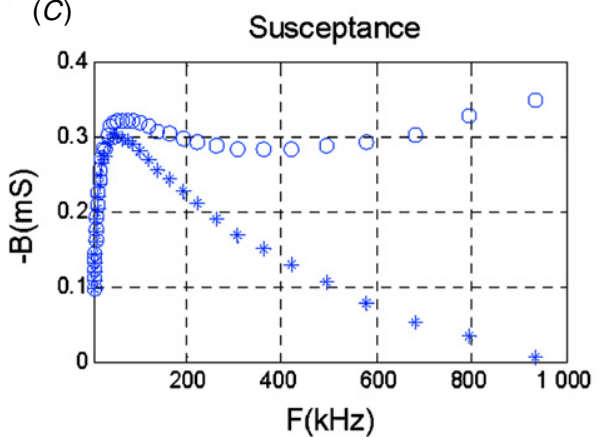

(B) Reactance

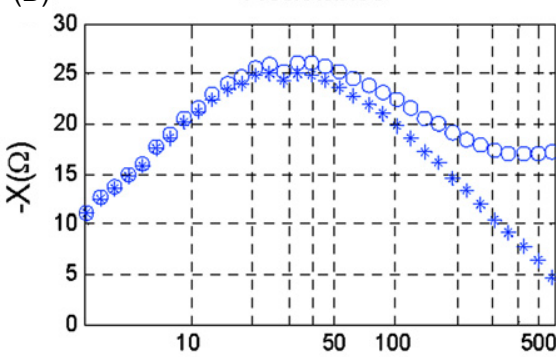

$\mathrm{F}(\mathrm{kHz})$

(D) Estimated Capacitance

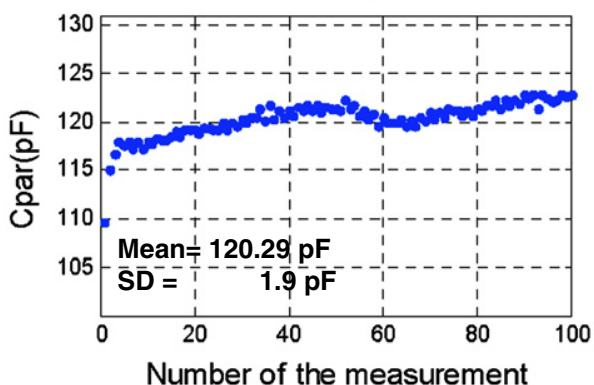

- Experimental Measurement $\quad * \quad$ Corrected Measurement $\quad$ Estimated Capacitance

Figure 7. Experimental results obtained from the segmental arm EBI measurements. Note that the mean and standard deviation values for the estimated capacitance are annotated in $(D)$. 

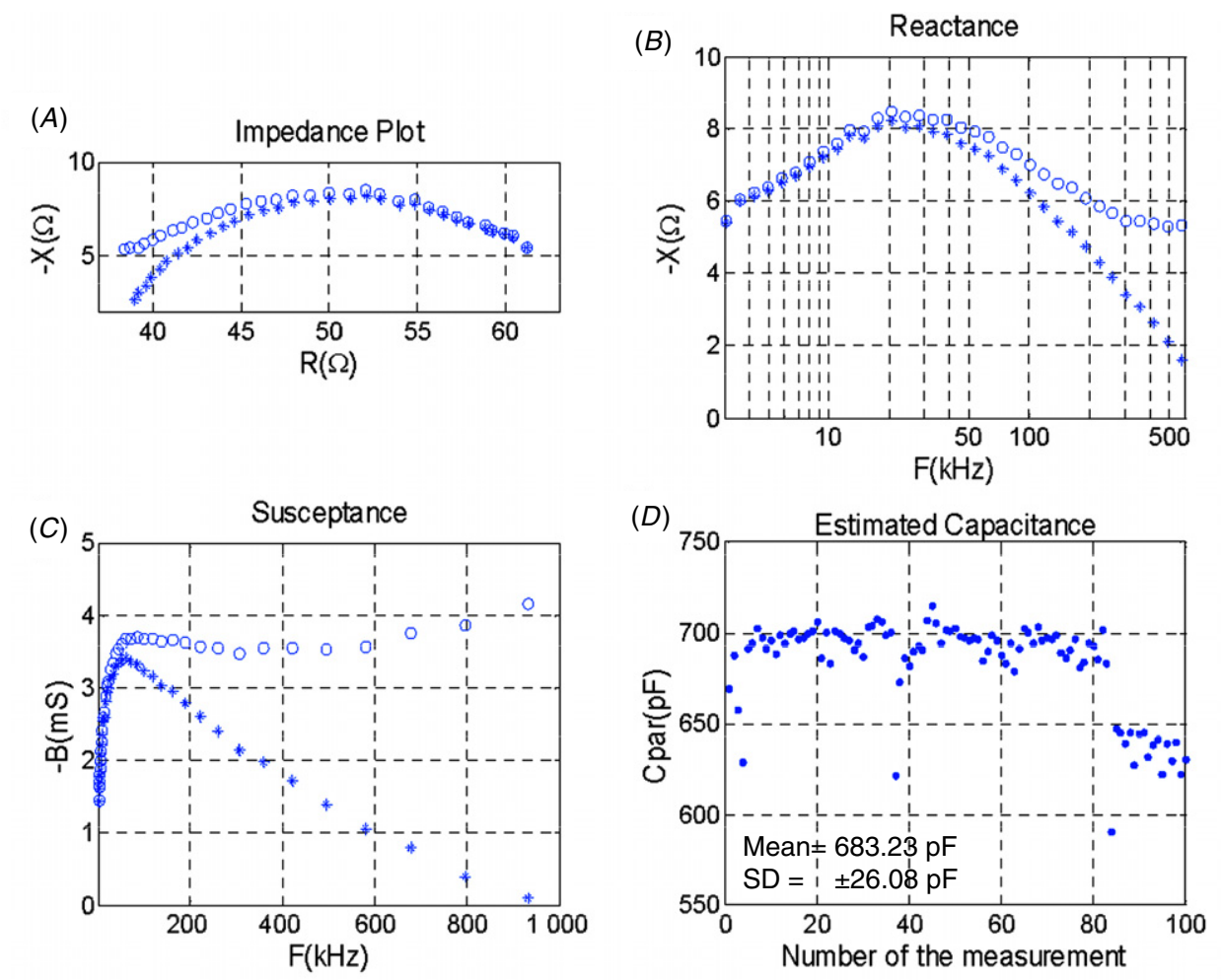

\section{- Experimental Measurement * Corrected Measurement - Estimated Capacitance}

Figure 8. Experimental results obtained from the segmental trunk EBI measurements. Note that the mean and standard deviation values for the estimated capacitance are annotated in $(D)$.

\section{Discussion and conclusion}

The experimental results shown here and the theoretical validation presented in Buendia (2009) and Buendia et al (2010) confirm that the proposed correcting method eliminates the hook-like deviation from the complex EBI data, correcting the effects of the capacitive leakage created by parasitic capacitances. The analysis presented here confirms that capacitive leakage alters all the EBI data-both the modulus and the phase of the EBI spectroscopy measurement-as previously reported by several authors (Bolton et al 1998, Buendia 2009, Buendia et al 2010, Scharfetter et al 1998). The experimental results presented here show that the proposed method completely corrects the data deviation observed in the complex impedance-both in the modulus and the phase. In this way, the mathematical intrinsic limitation of the $\mathrm{Td}$ compensation that prevents it from properly correcting the deviation is overcome.

This work confirms the idea proposed by Bolton et al (1998) that multiplying the measurements by a complex exponential with real scalar value, as is done with $\mathrm{Td}$ compensation, cannot correct the deviation observed in the EBI measurements. In this work, together with the theoretical validation presented in Buendia et al (2010), we have shown that for the Td compensation method presented in De Lorenzo et al (1997) to fully compensate the effects caused by capacitive leakage, Td must be complex and frequency dependent. When substituting Td by the proper expression in the complex exponential, the exponent disappears, leaving a correcting expression similar to the one proposed in Bolton et al (1998). The main difference between Bolton's correcting expression and the method proposed here is that the term Td is not just a real value but a complex function of frequency instead. This difference in the Td term from scalar to complex frequency function is the difference between compensation and correction.

A fundamental issue to address when evaluating the validity of the presented correction method is the origin of the data deviation observed at high frequencies. Two valid hypotheses have been formulated. One is that the data deviation results from the difference in time delays associated with the measurement channels, which is the basis of $\mathrm{Td}$ compensation. The other is that the data deviation results from capacitive leakage through parasitic capacitances associated with the measurement setup. Experimentally, the time delay difference hypothesis is unable to account for the deviation observed in the modulus of the impedance (Scharfetter et al 1998), while the capacitive leakage hypothesis is able to justify the deviation observed in both the modulus and the phase (Bolton et al 1998, Buendia 2009, Buendia et al 2010). From the theoretical point of view and to the knowledge of the authors, not a single electrical model has been proposed to validate the time delay difference theory as the source of the EBI data deviation observed at high frequencies. On the other hand, the presence of stray parasitic capacitances in EBI measurements setups is widely accepted and several authors, 
through simulations, mathematical modeling and experimental tests, have proposed such a parasitic capacitance as the source of the aforementioned EBI data deviation (Bolton et al 1998, Buendia 2009, Buendia et al 2010, Scharfetter et al 1998).

In any case, $\mathrm{Td}$ compensation has been used to compensate the high-frequency deviation for more than a decade because it does compensate in an effective manner, according to Scharfetter et al (1998). For pragmatic purposes and when planning on performing a post hoc Cole model-based analysis, the data compensation obtained by Td might be good enough. The main risk of using a single scalar value for Td is that full correction of the phase can only be achieved at a single frequency; above that frequency the phase data become overcompensated. Such overcompensation can influence post hoc Cole fitting procedures, especially when the fitting is done on the impedance plane, therefore producing incorrect estimations of the Cole parameters.

An important difference between the approach proposed in this work and the previous methods presented in De Lorenzo et al (1997) and Bolton et al (1998) is that the previous methods lack any procedure to select the value for the scalar Td that would compensate for the observed deviation in the EBI data. In this paper, in addition to the correcting expression presented in equation (5), a procedure to select the values of such an expression has been presented and successfully validated. From the practical point of view, it is precisely this procedure that makes the proposed method of correction especially significant for EBI measurement applications.

The main limitation of the proposed method resides in the difficult task of estimating the parasitic capacitance from the measured susceptance, which requires performing EBI measurements up to very high frequencies; theoretically the best estimation is done with measurements up to $\infty$. For single dominant dispersion systems, measuring up to frequencies above the main dispersion works very well. As long as an increasing slope can be detected on the susceptance spectrum, the estimation can be properly done. In this manner, when measuring immittance of biological tissue with more than a single dominant dispersion, as long as the measurement frequency range is wide enough to characterize the dominant dispersions, it should be possible to identify the increasing slope from the measured susceptance caused by prospective parasitic capacitances. In any case, this issue should be properly addressed to identify which EBI applications can benefit from the proposed correcting method.

Another limitation regarding the estimation of the parasitic capacitance from the measured susceptance is the fact that although the measurements may be influenced by capacitive leakage, which increases the susceptance spectrum at high frequencies, such an influence might not be enough to create an identifiable slope in the measured frequency range. In this case, it could be argued that the data deviation is so small that it would not affect any further data analysis, or perhaps it could be desirable to follow the recommendation of Scharfetter to not trust EBI measurements above a certain frequency, e.g. $500 \mathrm{kHz}$ (Scharfetter et al 1998). Note that the latter approach is only recommended when the influence of the capacitive leakage is insignificant; otherwise, the truncated impedance data will still contain deviations at lower frequencies. Another valid approach would be to make an iterative fitting of the obtained complex EBI data to a modified extended Cole model containing the correcting expression proposed in equation (5).

\section{References}

Aberg P, Geladi P, Nicander I, Hansson J, Holmgren U and Ollmar S 2005 Non-invasive and microinvasive electrical impedance spectra of skin cancer-a comparison between two techniques Skin Res. Technol. 11 281-6

Aberg P, Nicander I, Hansson J, Geladi P, Holmgren U and Ollmar S 2004 Skin cancer identification using multifrequency electrical impedance - a potential screening tool IEEE Trans. BioMed. Eng. 51 2097-102

Beckmann L, van Riesen D and Leonhardt S 2007 Optimal electrode placement and frequency range selection for the detection of lung water using Bioimpedance Spectroscopy Engineering in Medicine and Biology Society, 2007: EMBS 2007, 29th Ann. Int. Conf. IEEE (Lyon) pp 2685-8

Bolton M P et al 1998 Sources of error in bioimpedance spectroscopy Physiol. Meas. 19 235-45

Buendia R 2009 A novel approach for removing the Hook effect artefact from electrical bioimpedance spectroscopy measurements, University of Borås, Borås. Available from http://bada.hb.se/bitstream/2320/5418/1/Buendia.pdf

Buendia R, Seoane F and Gil-Pita R 2010 Novel approach for removing the hook effect artefact from electrical bioimpedance spectroscopy measurements J. Phys.: Conf. Ser. 224 121-6

Cole K S 1940 Permeability and impermeability of cell membranes for ions Quantum Biol. 8 110-22

De Lorenzo A, Andreoli A, Matthie J and Withers P 1997 Predicting body cell mass with bioimpedance by using theoretical methods: a technological review J. Appl. Physiol. 82 1542-58

Ivorra A 2005 Contributions to the measurement of electrical impedance for living tissue ischemia injury monitoring, Technical University of Calalonia, Barcelona. Available from http://www.tdr.cesca.es/TESIS_UPC/AVAILABLE/ TDX-0302105-135356//

Kyle U G et al 2004 Bioelectrical impedance analysis: part I. Review of principles and methods Clin. Nutr. 23 1226-43

Mirtaheri P, Grimnes S and Martinsen $\varnothing \mathrm{G} 2007$ Designing a $\mathrm{PtCO}_{2}$ sensor based on conductivity measurements 13 th Int. Conf. on Electrical Bioimpedance and 8th Conf. on Electrical Impedance Tomography (Graz), IFMBE Proc. 17 (9) 300-3

Mirtaheri P, Grimnes S, Martinsen Ø G and Tønnessen T I 2004 A new biomedical sensor for measuring $\mathrm{PCO}_{2}$ Physiol. Meas. 25 421-36

Moissl U M et al 2006 Body fluid volume determination via body composition spectroscopy in health and disease Physiol. Meas. 27 921-33

Scharfetter H, Hartinger P, Hinghofer-Szalkay H and Hutten H 1998 A model of artefacts produced by stray capacitance during whole body or segmental bioimpedance spectroscopy Physiol. Meas. 19 247-61

Schwan H P 1957 Electrical properties of tissue and cell suspensions Adv. Biol. Med. Phys. 5 147-209

van Marken Lichtenbelt W D, Westerterp K R, Wouters L and Luijendijk S C 1994 Validation of bioelectrical-impedance measurements as a method to estimate body-water compartments Am. J. Clin. Nutr. $60159-66$ 